\title{
Le bon usage du médicament : définition, référentiels, périmètre et champ d'application
}

\author{
Les participants à la table ronde $n^{\circ} 2$ de Giens XXIII ${ }^{\star}$
}

Texte reçu le 8 juillet 2008 ; accepté le 30 juillet 2008

\begin{abstract}
Mots clés :
bon usage ; groupe homogène de séjour; médicaments : référentiels
\end{abstract}

\begin{abstract}
Résumé - Le bon usage des médicaments peut être défini simplement comme l'utilisation du bon médicament, à la bonne dose, pendant la durée nécessaire, pour un patient donné qui le tolère correctement.

Il est impossible, étant donné le nombre de médicaments et le nombre infini des situations cliniques individuelles de ne pas baser la prescription sur une référence. La référence peut être unique, lorsque la situation clinique est simple et le médicament, à choisir, unique. Dans ce cas une bonne formation initiale et continue sert de référence au choix. L'usage du RCP (Résumé des Caractéristiques du Produit) choisi permet d'obtenir une prescription plus précise et plus sûre. Dans beaucoup de cas l'usage de telle ou telle stratégie thérapeutique peut conduire au résultat souhaité. Dans ces cas, des référentiels scientifiques permettent d'aider le prescripteur à choisir la meilleure stratégie. Enfin les stratégies thérapeutiques peuvent avoir une efficacité égale et des coûts très différents. Des référentiels peuvent être émis pour diminuer le coût d'une prescription donnée.

Les difficultés issues de références ou de référentiels sont :

1. Ils ne peuvent pas couvrir l'intégralité des situations cliniques.

2. Ils devraient être évolutifs à mesure que la science progresse et cela est difficile.

3. La multiplicité des émetteurs aboutit à des messages confus parfois contradictoires.

4. Leur pertinence et leur efficacité devraient être évaluées.

5. Ils peuvent être inutilisables pour le prescripteur en raison de leur complexité ou de l'éloignement des situations pratiques. Nous proposons un constat et un certain nombre de solutions pour améliorer la qualité et la pertinence des références ou référentiels : des structures de coordination choisissant les thèmes et définissant les méthodes de rédaction, la création d'un répertoire des référentiels, un cahier des charges de tout référentiel avec exigence de qualité, une formation initiale et continue des soignants destinataires des référentiels. Il est par ailleurs nécessaire de mettre en place des mesures d'impact de ces référentiels et de s'imposer une actualisation adaptée périodique. Tout cela devrait permettre une amélioration mesurable de la qualité des soins.
\end{abstract}

Le bon usage des médicaments fait partie intégrante d'une bonne pratique médicale. On le définit d'une façon volontairement simpliste comme l'utilisation du bon médicament, à la bonne dose, pendant la durée nécessaire, à un patient qui le tolère bien.

Le résultat d'un traitement est également conditionné par une prescription respectueuse des règles en usage dans les hôpitaux ou en ville, par une dispensation correcte et par le fait que le malade suive bien son traitement. Nous écarterons ces aspects qui s'éloignent du sujet de la table ronde de Giens 2007 qui était centrée sur les références et référentiels sous-tendant le choix d'une stratégie thérapeutique.

\footnotetext{
* Pour la liste des participants, voir en fin d'article.
}

\section{La notion de médecine fondée sur les preuves et les référentiels scientifiques}

La multiplication et le perfectionnement des essais thérapeutiques ont fait apparaître une nouvelle tentative de rationalisation de la médecine.

Celle-ci repose sur l'idée que le choix d'une thérapeutique donnée, doit prendre en compte ce que la science a démontré. Ce modèle est très réducteur. Le concept complet est que : le choix d'un traitement donné, pour un patient donné, par un médecin donné doit prendre en compte ce que la science a démontré, ce que le médecin sait par expérience et à propos du malade concerné et ce que le malade est prêt à accepter. Il s'agit donc 
bien de l'adaptation à un patient unique, des données scientifiques pondérées par l'expérience du médecin.

Les limites de ce modèle viennent de l'abondance des sources scientifiques et des difficultés de leur interprétation d'une part et d'autre part du fait qu'un malade donné est rarement absolument comparable à ceux qui ont été inclus dans les essais thérapeutiques.

En pratique, c'est la nécessité d'intégrer et de pondérer les données de la science qui aboutit à l'écriture de la majorité des référentiels de bon usage du médicament intégrant ces données scientifiques.

Les piliers de la prescription basée sur ces référentiels sont : l'objectivité des référentiels, leur fiabilité scientifique, et l'adaptation au patient donné. Comme nous allons le voir ci-dessous la précision de la prescription d'un produit choisi passe par une vérification de nos connaissances et par la lecture du RCP (résumé des caractéristiques du produit).

\section{Les cas les plus simples}

Le plus souvent le bon usage des médicaments découle d'une bonne connaissance du traitement d'une affection donnée, acquise en formation initiale ou continue. En cas de doute quant aux modalités du traitement, le recours au RCP du médicament choisi permettra une prescription plus précise en terme de dose, de durée, d'adaptation à l'âge, à la fonction rénale ou hépatique. De même la lecture du RCP permet une prescription plus sûre en précisant les effets indésirables, leur fréquence et les interactions médicamenteuses.

Ces situations sont nombreuses. Par exemple, lorsque le diagnostic d'hypothyroïdie a été posé et que sa cause a été précisée, le traitement de substitution par la thyroxine est simple et la surveillance aisée sur la base des signes cliniques et d'un dosage biologique unique et fiable.

En fonction de la palette de connaissances d'un médecin, le nombre de situations où la prescription est ainsi presque automatique est plus ou moins grand.

Le diagnostic d'œdème pulmonaire chez un insuffisant cardiaque conduira pratiquement toujours à la prescription de diurétiques de l'anse. Au décours de cet épisode, l'initiation ou l'adaptation du traitement par les inhibiteurs de l'enzyme de conversion relève plus de la question de cours que de la lecture d'un référentiel compliqué.

Cette médecine automatique a une limite importante : l'évolution des connaissances. Un exemple est celui du traitement des angines : depuis le recours presque systématique aux pénicillines, qui a eu le mérite de participer à la disparition de maladies comme le rhumatisme articulaire aigu ou la glomérulonéphrite post-streptococcique, jusqu'à la campagne intitulée « les antibiotiques c'est pas automatique », on a tout simplement changé d'urgence épidémiologique : prévention de maladies graves dans les années 60 , prévention de la résistance aux antibiotiques aujourd'hui.

La médecine évolue. Les bêtabloquants, jadis honnis, dans l'insuffisance cardiaque font aujourd'hui partie du traitement de base de la plupart des formes de cette maladie. Le bon usage du médicament, dans les situations les moins discutables, peut évoluer.

Les piliers de ce bon usage de tous les jours sont la formation initiale, la formation continue, l'évaluation entre pairs de la pratique professionnelle et le recours aussi souvent que nécessaire à la lecture du RCP des produits qu'on prescrit.

\section{L'intégration des données économiques}

Le budget alloué aux dépenses de médicaments, et aux dépenses des différents établissements de soins est un «ensemble fini » dont le taux de croissance est défini chaque année par la loi.

L'apparition de nouvelles thérapeutiques ou l'extension des indications de certains produits amène à de nouvelles dépenses qui doivent nécessairement s'accompagner d'un encadrement des nouvelles prescriptions et d'une diminution des coûts de traitements plus anciens.

L'encadrement des prescriptions peut procéder :

1. De mesures autoritaires comme les déremboursements ou l'incitation à la prescription de génériques. Ces mesures ont un fondement scientifique et leur réalité s'impose au soignant.

2. De mesures résultant d'un accord entre prescripteurs et payeurs comme les accords de bon usage.

\section{Les références ou référentiels : principes de rédaction}

Nous avons vu qu'un référentiel peut être édité pour des raisons diverses. Le médecin peut dire : je me suis référé, pour choisir le produit, à telle notion que j'ai apprise en formation initiale ou continue, ou à tel référentiel établi par une instance scientifique que je reconnais ou à tel accord passé entre les payeurs et les médecins. Dans tous les cas, j'ai adapté ma prescription au patient donné en me basant sur ce qui le rend unique et ce que contient le $\mathrm{RCP}$ et les autres textes réglementaires concernant le produit.

Les objectifs des émetteurs de référentiels sont donc divers : éducation, rationalisation, précision, intégration de données scientifiques nombreuses, intégration de données économiques. Ces objectifs divers aboutissent à une multiplicité des émetteurs de 
référentiels qui considèrent, tous et chacun à juste titre, avoir une légitimité.

\section{1. Les référentiels de base pour un produit donné}

L'acte de base dans la vie d'un médicament a longtemps été l'obtention d'une autorisation de mise sur le marché (AMM) et l'écriture d'un RCP. Cet acte est toujours indispensable mais est maintenant un extrait de naissance auquel viennent s'ajouter un plan de gestion de risque, un plan de minimisation des risques, un avis de transparence et une éventuelle admission aux remboursements. Les trois instances en charge : l'Afssaps (Agence Française de Sécurité Sanitaire des Produits de Santé), la HAS (Haute Autorité de Santé) et le Comité Économique des Produits de Santé, peuvent demander des études complémentaires visant à préciser les conditions d'utilisation dans des populations particulières (enfants, sujets âgés), les contours épidémiologiques de la population concernée par le traitement en France et la quantification plus précise du bénéfice apporté par une nouvelle thérapeutique.

Tous ces éléments complètent l'information sur un produit donné et forment le référentiel d'usage d'un produit donné.

Ces informations sont évolutives en fonction des données scientifiques positives ou négatives qui jalonnent la vie d'un produit ou d'une classe de produit.

Deux exemples opposés illustrent ce phénomène : l'extension d'indication de l'herceptine dans le cancer du sein et la limitation des indications des médicaments utilisés en traitement de la ménopause.

Dans les deux cas une information scientifique nouvelle a mené à une auto saisine de la commission d'AMM pour aboutir rapidement dans le premier cas, plus difficilement dans le deuxième, à une modification radicale du RCP d'un produit ou d'une classe de produits.

Tous les changements ne sont pas aussi manifestes et rapidement connus. C'est l'un des grands problèmes de «l'émetteur Afssaps » : les modifications mineures, modestes, mais parfois importantes du RCP des produits, le renforcement ou la levée d'une précaution d'emploi, la modification des conditions de distribution d'un produit, l'harmonisation des RCP à l'échelle de l'Europe ou à l'intérieur d'une classe de produits passent souvent inaperçus du prescripteur. Ces changements n'en demandent pas moins, beaucoup de travail de la part des firmes et de la part des autorités d'enregistrement; le processus est souvent très long.

La nouvelle scientifique tend à précéder de loin le changement du RCP et d'encore plus loin l'actualisation des référentiels.

Néanmoins le RCP est une des bases d'établissement des référentiels dans la grande majorité des cas.

\subsection{Référentiels de bon usage basés sur des données scientifiques}

Leur établissement suit un protocole désormais bien défini.

Un groupe restreint réunit toutes les informations scientifiques disponibles. Un groupe d'écriture cherche à intégrer et pondérer ces informations pour aboutir à la description d'une stratégie thérapeutique adaptée à la question médicale traitée. Un groupe de lecture a pour mission de critiquer, compléter les informations et de s'assurer que la part faite à l'exhaustivité ne nuit ni à la compréhension ni à l'utilisation des recommandations par les praticiens concernés.

Ainsi, le référentiel est souvent scindé en deux parties. Le référentiel proprement dit, court et applicable aux différentes situations cliniques concernées d'une part et sa justification scientifique exhaustive, nécessairement longue pour toutes les pathologies un tant soit peu complexes d'autre part.

\subsection{Référentiels de bon usage prenant en compte des données économiques}

Ils deviennent nécessaires dans deux circonstances :

- Lorsqu'une pathologie donnée peut être traitée avec une égale efficacité par des produits ayant un coût très différent.

- Et lorsqu'un médicament apporte un progrès médical important pour un petit nombre de patients, mais à un prix très élevé.

Les autorités sont alors confrontées à un double problème, obtenir que ce traitement soit utilisé dans les limites étroites de son AMM et ne pas déséquilibrer le budget d'un hôpital au motif qu'il concentre les patients relevant de ce nouveau traitement. Un accord est alors passé pour que le budget nécessaire soit ajouté au coût habituel de prise en charge d'une pathologie donnée. Cet accord est conditionné par le fait que les médecins qui soignent ces patients puissent démontrer que le traitement n'est administré qu'à des malades relevant clairement de son utilisation.

\subsection{Contrats de Bon Usage pour les médicaments innovants et coûteux}

Certains médicaments innovants et coûteux sont facturés en sus et sont donc hors du groupe homogène de séjour (GHS). Pour ces médicaments et dispositifs médicaux stériles implantables, des référentiels de bon usage (RBU) créés par des structures régionales sont validés par l'Afssaps, l'HAS et l'INCa (Institut National du Cancer). Elles définissent pour chacun de ces produits quatre catégories :

I. Indications reconnues (AMM). 
II. Indications pertinentes, acceptables (hors AMM mais avec un haut niveau de preuve d'efficacité et de rapport bénéfice/risque favorable).

III. Situations contre-indiquées non acceptables (existence d'études prouvant l'absence d'efficacité et/ou le rapport bénéfice/risque défavorable).

IV. Situations autres pour lesquelles l'insuffisance de données ne permet pas d'évaluer le rapport bénéfice/risque : le traitement n'est ni recommandé, ni contre-indiqué (quelques cas cliniques ou quelques données laissent préjuger d'une efficacité sans démonstration à haut niveau de preuve, voire utilisation compassionnelle légitime).

Pour ces médicaments qui couvrent de nombreuses situations hospitalières, notamment en cancérologie, dans les maladies rares et orphelines, dans les pathologies immunologiques sévères, il est demandé aux prescripteurs de préciser systématiquement l'indication justifiant de l'usage du médicament. En cas de prescription abusive du niveau III ou IV, des sanctions financières doivent être envisagées.

\section{Difficultés concernant l'établissement et l'application des référentiels}

Nous avons dit plus haut les difficultés que peuvent rencontrer les émetteurs primaires (Afssaps, HAS) pour faire connaître les modifications des informations constituant la référence d'utilisation d'un produit donné. Ces difficultés sont multipliées à l'infini pour ce qui concerne l'établissement de référentiels basés sur des données scientifiques.

\section{1. Le choix du sujet}

Toutes les pathologies ne se prêtent pas à l'écriture d'un référentiel de bon usage des thérapeutiques. Nous l'avons dit des pathologies simples, mais cela est également vrai des pathologies dans lesquelles le traitement est rapidement évolutif ou, à l'inverse de pathologies dans lesquelles le traitement est peu efficace.

Le choix du sujet dépend en outre de l'émetteur du référentiel et de ses motivations.

\subsection{La multiplicité des émetteurs de référentiels et l'inégalité devant l'élaboration}

Lorsqu'une société savante choisit un sujet pour écrire un consensus ou un référentiel, ses contraintes et objectifs ne sont pas les mêmes que lorsque les autorités sanitaires entreprennent la même démarche.
L'objectif de la société savante sera d'aboutir à une publication scientifique, ayant le plus grand retentissement possible et qui sera citée en référence dans les articles consacrés à ce sujet ultérieurement. Il en va ainsi des recommandations pour les traitements de l'hypertension artérielle. Périodiquement, aux ÉtatsUnis, le Joint National Committee publie un consensus; les Européens ne sont pas en reste et publient leurs recommandations pour le traitement de l'hypertension artérielle. Ils bénéficient d'un processus d'édition et d'illustration du référentiel mis à leur disposition par le journal qui a également intérêt à ce que le texte soit repris le plus souvent possible. Les auteurs mandatés par la Société Savante se basent explicitement sur les données scientifiques en s'affranchissant plus ou moins du contenu des RCP des produits cités. Les référentiels des sociétés savantes peuvent être écrits à l'intention d'un petit nombre de praticiens ayant une pratique très spécialisée : référentiels établis par l'institut du Cancer, référentiels d'usage et de pratique de l'angioplastie coronaire par exemple.

À l'inverse, les référentiels élaborés par les autorités sanitaires ont pour sujet le traitement de pathologies fréquentes ou l'utilisation de médicaments très largement prescrits. Dans ces référentiels, les données scientifiques sont à égalité avec les données contenues dans le RCP des produits. Un même émetteur peut difficilement écrire des choses contradictoires entre les RCP et les référentiels. De même le souci d'applicabilité est central dans ces référentiels. Les référentiels sur des sujets très spécialisés sont écrits par des spécialistes de la pathologie traitée à l'intention des autres spécialistes de cette discipline. Le texte ainsi établi a de grandes chances d'être compris et d'être applicable.

À l'inverse, dans les référentiels portant sur des pathologies très fréquentes et/ou des classes de produits largement utilisées, les références de bon usage sont établies par des spécialistes; même lorsque les groupes d'écriture et de lecture comportent des médecins exerçant en médecine générale, il s’agit souvent de médecins plus intéressés voire plus instruits que la majorité de leurs confrères. Ainsi, le texte final peut être difficile à utiliser pour la pratique sauf si le référentiel court est axé sur la pratique et s'il est soumis à un test de lecture par des médecins tirés au sort.

Enfin les référentiels commandés par l'Afssaps ou par la HAS bénéficient encore trop peu d'un processus d'édition les rendant plus aisément compréhensibles.

\subsection{L'évolutivité}

L'écriture d'un RCP, d'un référentiel de bon usage ou même d'un accord de bon usage basé sur des notions économiques, a l'inconvénient de figer l'image à un instant « $\mathrm{t}$ ». Les événements scientifiques ultérieurs vont modifier ce que l'on sait d'un produit donné, pour une indication donnée. Mais le processus qui conduit 
à une modification du RCP ou de l'avis de transparence est d'une grande lenteur.

Autant cette lenteur est justifiée pour la première inscription, autant, elle est un poids très lourd pour l'évolution des références de base. Ainsi l'AMM peut être dépassée par la réalité. Dans les mois qui ont suivi la commercialisation du clopidogrel, les praticiens de l'angioplastie coronaire, qui avaient déjà établi l'utilité de la ticlopidine dans la prévention de l'occlusion des stents bien avant que le RCP de ce produit en fasse mention, sont passés de la ticlopidine au clopidogrel pour éviter les agranulocytoses liées au premier produit. Le RCP du clopidogrel ne porte mention de cette utilisation quasiment universelle que depuis peu.

Seuls, les changements des données économiques peuvent aboutir parfois à un changement d'accord entre les praticiens et les payeurs. L'exemple des statines au Royaume-Uni est instructif à cet égard : ce pays a longtemps été un faible consommateur de statines alors même que la prévalence de l'athérosclérose coronaire y est importante. Depuis que la simvastatine est génériquée, les autorités encouragent une utilisation très large de ce produit devenu moins onéreux et de fait la Grande-Bretagne a rattrapé le peloton de tête des pays consommateurs de statines en Europe.

\subsection{L'évaluation}

Rédiger un référentiel est un processus long et complexe qui mobilise un grand nombre d'acteurs. Il est donc naturel d'en évaluer l'efficacité. Celle-ci ne peut être mesurée que par la fréquence d'adoption de comportements thérapeutiques adéquats pour un type de patient donné.

Dans certains pays où la constitution de cohortes de patients est aisée de par l'existence d'une informatisation des données médicales de la population entière ou d'une partie seulement de la population, on peut mesurer la fréquence de prescription de bêtabloquants à des malades ayant eu un infarctus du myocarde. Ces études permettent également de mesurer la différence de pronostic entre les patients recevant une prescription suivant les référentiels et les autres.

Dans les pays ne disposant pas d'une informatisation systématique, des enquêtes, faites pendant une période de temps donné, permettent d'évaluer l'atteinte des objectifs fixés par les référentiels. Ces registres sont souvent financés par l'industrie pharmaceutique en totalité ou en complément d'argent public. La répétition de ces enquêtes dans le temps permet de mesurer l'évolution de l'adhésion aux référentiels.

Le principe de base de ces évaluations est de pouvoir croiser des données thérapeutiques et diagnostiques fiables. La France souffre d'un déficit considérable en la matière. Les données informatisées saisies par les caisses d'assurance maladie sont rarement utilisées dans le but d'évaluer l'atteinte d'un optimum thérapeutique.

\section{Propositions de solutions}

Les thèmes des référentiels devraient naître d'une observation constante de la pratique médicale. Lorsqu'une pathologie est fréquente ou grave ou coûteuse, lorsqu' on identifie une forte hétérogénéité des pratiques ou a fortiori un mésusage patent, l'écriture d'un référentiel ou le renforcement des conseils d'utilisation d'un produit sont nécessaires. L'autre source devrait être l'apparition d'une donnée scientifique importante justifiant un ajustement rapide des pratiques.

L'élaboration d'un référentiel doit être faite en ayant surtout le souci de l'utilisateur et se heurte à la multiplicité des émetteurs de référentiels. Il serait souhaitable qu'une coordination entre autorités réglementaires et sociétés savantes soit mise en place pour éviter la publication de référentiels trop redondants ou trop discordants. Lorsqu'un groupe, comme celui qui édite périodiquement les recommandations d'usage des antithrombotiques, le fait avec une exhaustivité totale et en libellant ses conseils par niveau de preuve, il peut paraître vain d'écrire un référentiel émanant des autorités réglementaires. On pourrait imaginer que lorsqu'un référentiel étranger objectif et de bonne qualité est publié, un groupe d'expert français en fasse une synthèse de lecture aisée tout en laissant aux autorités réglementaires la possibilité de faire état de particularités françaises. Un référentiel, quel qu'il soit, ne devrait pas pouvoir être publié sans qu'un ou plusieurs groupes comportant des médecins libéraux généralistes ou spécialistes aient exprimé leur avis quant à la lisibilité et à la possibilité d'appliquer le référentiel en question.

L'application d'un référentiel nécessite une diffusion ciblée vers les prescripteurs concernés et une stratégie concertée d'exploitation de ce texte. Il est nécessaire de réfléchir à la façon dont le prescripteur va s'approprier ce référentiel et il faut mettre en place des relais d'information (associations de patients, grande presse...). Il faut développer les aides dématérialisées pour les professionnels de santé et imposer des règles de diffusion identiques pour tous, qu'il s'agisse de l'industrie ou des caisses d'assurance maladie. Il est légitime d'utiliser la FMC (Formation Médicale Continue) comme outil d'appropriation et il est nécessaire de suivre l'application des référentiels grâce à l'évaluation des pratiques professionnelles. Les prescripteurs doivent être incités à justifier leurs choix lorsqu'ils diffèrent de ceux des recommandations. Enfin, on peut impliquer le patient en développant des programmes spécifiques. Toutes ces propositions pouvant inciter à respecter les référentiels, tant au niveau des professionnels de santé que des patients, seraient au mieux définies dans une charte de diffusion de l'information. 
L'évaluation d'un référentiel expose à la multiplicité des outils, des méthodes et des points de vue. Aucune des évaluations, qu'elles soient diligentées par les industriels, la CNAM (Caisse Nationale d'Assurance Maladie) ou des instances scientifiques n'est incontestable. Il est donc indispensable qu'une méthode solide d'évaluation soit définie en même temps que le référentiel est publié. Là encore une coordination entre les parties prenantes sur des mesures simples d'atteinte des objectifs thérapeutiques est nécessaire. Une pédagogie d'acceptabilité d'un nouveau référentiel doit être mise en place utilisant la formation médicale continue et l'évaluation des pratiques professionnelles. Il pourrait ainsi être proposé de mesurer le pourcentage d'adéquation au référentiel dans le cadre d'accords cadres, ou d'évaluation des pratiques professionnelles, ou d'accréditation par l'HAS. Une étude postAMM pourrait aussi permettre d'améliorer l'effet d'un référentiel sur les prescriptions dans le cadre d'études de recherche sur les pratiques.

L'actualisation d'un référentiel est aujourd'hui le parent pauvre de cette pratique. Il devrait pourtant être possible de publier grâce à l'informatique des informations destinées au prescripteur et prenant en compte les résultats des études demandés par l'Afssaps ou par la HAS, les résultats des études scientifiques marquant une évolution importante dans un sens restrictif ou d'élargissement devraient pouvoir être examinés d'autant plus rapidement que l'enjeu de santé publique est plus important. D'une façon pratique un référentiel ne devrait être considéré comme valide que dans les 5 à 7 années suivant son écriture. La veille scientifique qu'elle vienne des professionnels, de la DGS (Direction Générale de la Santé), de la CNAM, des agences, de la pharmacovigilance ou des industriels devrait être le point de départ de ces actualisations. Aujourd'hui ces actualisations sont trop souvent retardées par des intérêts divergents. Cette actualisation impose donc coordination, hiérarchisation, priorisation et regroupement des efforts.

\section{Conclusions}

L'impression retirée de cette analyse de la conception, de la diffusion, de l'évaluation et de l'actualisation des référentiels est que l'idéal serait qu'une autorité médico-scientifique écrive les recommandations à la demande et dans l'intérêt des prescripteurs et des autorités. Ceci impose une cellule de coordination et un répertoire des référentiels. Une exigence de qualité est absolument nécessaire. Il est donc important qu'une autorité centrale partant des référentiels existants d'une part et de la pratique d'autre part s'appuie sur un émetteur unique des référentiels destinés à améliorer les pratiques. Ceci ne s'oppose pas à la nécessité de sources multiples transversales et complémentaires pour l'élaboration des référentiels. Aucun référentiel ne devrait être publié sans une formation des utilisateurs et sans possibilité d'évaluation de son impact sur l'adéquation de la prescription et quant à son influence sur la santé publique.

\section{Participants}

Michel Andrejak (CHU, Amiens), Jean-Pierre Bader (Hôpital Fernand Vidal, Paris), Marion Bamberger (Bristol Myers Squibb), Jean-François Bergmann (Hôpital Lariboisière, Paris), Pascal Bilbault (Boehringer), Régis Bordet (CHU, Lille), Agnès Brouard (AGEPS), Anne Burstin (Afssaps), Alain Castaigne (AstraZeneca), Anne Castot (Afssaps), Alain Coulomb (Consultant, Paris), Muriel Dahan (Institut National du Cancer [INCa]), Frédéric DeBels (Haute Autorité de Santé [HAS]), Corinne Duguay (Sanofi Aventis), Nathalie Dumarcet (Afssaps), Pierre Fender (Caisse Nationale de l'Assurance Maladie [CNAM]), Christian Funck Brentano (Hôpital Saint Antoine, Paris); Isabelle Giri (Consultant, Verrières-le-Buisson), Pascale Jolliet (CHU, Nantes), Murielle Jousselon Pautrot (Afssaps), Florence Leclerc (Roche), Patricia Le Gonidec (Agence Régionale de l'Hospitalisation, Île de France [ARHIF]), Patricia Maillere (Iris), François Meyer (Haute Autorité de Santé), Thierry Moreau Defarges (Janssen Cilag), Olivier Obrecht (HAS), Gilles Paintaud (CHU, Tours), Nicole Petitcollot (Direction Générale de la Santé [DGS]), Marc Samama (Hôpital Hôtel Dieu, Paris), Julia Sauterey (Observatoire des Médicaments des Dispositifs médicaux et des Innovations Thérapeutiques Île de France [OMEDIT IDF]), Pierre Schiavi (Servier), Philippe Tcheng (Sanofi Aventis), Patrick Villani (AP HM Hôpitaux de Marseille), Monique Weber (Caisse Nationale de l'Assurance Maladie des Travailleurs Salariés [CNAMTS]), Nadine Weisslinger Darmon (GlaxoSmithKline), Myriam Zylberman (Lilly).

\section{For more informations}

Décret n²005-1023 du 24 août 2005 relatif au « contrat de bon usage des médicaments et des produits et prestations », mentionné à l'article L.162-22-7 du code de la sécurité sociale

Méthodologie générale d'élaboration des protocoles thérapeutiques «hors-GHS »: http: //agmed.sante.gouv. fr/htm/3/t2a/html/methodologie.pdf

Accord de bon usage des soins relatif à l'utilisation des antiagrégants plaquettaires JO 5 janvier 2007

Accord-cadre national relatif au bon usage des antibiotiques dans les établissements de santé J.O n 150 du 29 juin 2002 page 11215 http://www . antibiotiquespasautomatiques.com/

Fiches de bon usage du médicament (BUM) HAS http://www . has-sante.fr/ portail/display.jsp?id=c_412202

Recommandations bonne pratique (RBP) Afssaps-HAS http://agmed.sante gouv. fr/htm/5/rbp/indrbp.htm 
Les plans de gestion de risque http://agmed. sante.gouv.fr/htm/10/pgr/ indpgr.htm

Mise au point actualisée de juin $2006 \mathrm{du}$ traitement hormonal substitutif de la ménopause. http://afssaps.sante.fr//htm/10/ths/thm_mise_ aupoint.pdf

Recommandations en hypertension artérielle et médecine cardiovasculaire. http://www.sfhta.org/pages/recos_hta.html : 14 versions nationales et internationales (1997-2008)

Gusssous I, Rodondi N, Paccaud F. Accès sans prescription aux statines : bénéfique ou délétère ? Médecine de premier secours Revue médicale suisse 2005, 38: $2447-52$
Comment améliorer la qualité de l'antibiothérapie dans les établissements de soins ? Qualité : préserver l'intérêt collectif sans nuire à l'intérêt individuel. SPILF. Presse Med 2002; 31 (26): 1231-39

Correspondance et offprints : Jean-François Bergmann, Service de Médecine Interne A, Hôpital Lariboisière, 2 rue Ambroise Paré, 75010 Paris, France. E-mail : jf.bergmann@1rb.aphp.fr 